\title{
Nasopancreatic Drainage for 4 Patients during the Early Phase of Acute Pancreatitis
}

\author{
Morihisa Hirota Akinobu Koiwai Atsuko Takasu Keita Kawamura \\ Ryo Kin Katsuya Endo Takayuki Kogure Takayoshi Meguro \\ Kennichi Satoh \\ Division of Gastroenterology, Tohoku Medical and Pharmaceutical University, Sendai, Japan
}

\section{Keywords}

Acute pancreatitis - Endoscopic retrograde cholangiopancreatography - Endoscopic

treatment

\begin{abstract}
We encountered 4 patients with acute pancreatitis (AP) of various etiologies and coexisting acute cholangitis who underwent endoscopic biliary stenting (EBS) and nasopancreatic drainage (NPD) via endoscopic retrograde cholangiopancreatography (ERCP) during the early phase of AP. ERCP is performed to treat acute cholangitis even in the context of AP. However, in difficult cases, accidental contrast media injection or guidewire insertion into the pancreatic duct can happen during ERCP for the purpose of EBS. It is concerned that cannulation injury and increased pancreatic duct pressure can exacerbate existing AP. Because pancreatic guidewire-associated techniques were required for all of them due to difficult biliary cannulation, we performed a NPD catheter placement using the pancreatic guidewire to decompress the pancreatic duct to prevent further exacerbating AP. Surprisingly, all patients dramatically improved without systemic or local complications. NPD could be performed without any adverse events and did not worsen the course of AP. Early decompression of a pancreatic duct using NPD may rather improve AP that had already developed. Further prospective research is needed to confirm our observations.
\end{abstract}




\section{Introduction}

Endoscopic retrograde cholangiopancreatography (ERCP) is generally contraindicated for patients with acute pancreatitis (AP) because the procedure might exacerbate AP. However, for gallstone-induced biliary AP with acute cholangitis, practice guidelines state that ERCP is indicated to resolve the bile duct obstruction and drain infected bile [1,2]. According to practice guidelines for acute cholangitis, ERCP should be selected as the firstline therapy [3]. Thus, ERCP is performed to treat acute cholangitis even in the context of AP, even though ERCP remains technically challenging [4]. Accidental cannulation or guidewire insertion into the pancreatic duct can happen during ERCP for the purpose of endoscopic biliary stenting (EBS).

In such difficult cases, pancreatic guidewire (PGW)-assisted biliary cannulation is recommended [5]. PGW-assisted biliary cannulation is a technique where a guidewire is left in the main pancreatic duct while attempting to cannulate the biliary duct. This technique has a high success rate of biliary cannulation. Subsequent prophylactic pancreatic stenting has been shown to effectively prevent post-ERCP pancreatitis (PEP) [5]. Despite its usefulness in preventing PEP, adverse events of prophylactic pancreatic stenting such as infection, stent migration, and occlusion have been indicated [6]. In addition, the efficacy of pancreatic stenting for patients who have already developed AP has not been clarified [7]. Endoscopic nasopancreatic drainage (NPD) has been clinically used to treat pancreatic fistulas and diagnose early pancreatic cancer [8,9]. NPD is expected to decompress the pancreatic duct by draining pancreatic juice, just like stenting. Increased pancreatic duct pressure could exacerbate AP, and decompression could be effective for treating AP [10,11]. However, the efficacy of NPD for the early phase of AP has also not been clarified in a clinical setting. Moreover, there is even concern that NPD, which directly stimulates the pancreas, may exacerbate existing AP.

Here, we describe 4 patients with AP of various etiologies, including 1 case of alcoholic pancreatitis, 1 case of PEP, and 2 cases of biliary pancreatitis. The patients underwent ERCP for the purpose of EBS placement to treat coexisting acute cholangitis. Because PGW-associated techniques were required due to difficult biliary cannulation, NPD in addition to EBS was performed using the PGW. The patients experienced surprising rapid clinical improvement without worsening of AP.

\section{Case Report}

We treated 4 patients with AP who underwent urgent ERCP due to concomitant acute cholangitis. Characteristics of them are described in Table 1. EBS and NPD were both performed. PGW-associated techniques were required for all of them due to difficult biliary cannulation. Cannulation injury or passed biliary stone can cause edema of the ampulla of Vater (Fig. 1a). Since increased pancreatic duct pressure due to pancreatic duct obstruction resulting from edema at the ampulla of Vater is thought to worsen AP, we decided to perform NPD, instead of placing a pancreatic stent, using the PGW. The reason for choosing NPD was that a prophylactic pancreatic stent was thought to be associated with higher risk of infection. NPD has low risk of retrograde gut bacterial infection, unlike stent placement. The NPD catheter does not migrate into the pancreatic duct. The certainty of drainage with NPD is guaranteed because the drainage of pancreatic juice can be observed. Therefore, if nasopancreatic drain obstruction occurs, it is immediately known. The cause of obstruction can be identified and resolved in many cases. NPD has the disadvantage of causing discomfort in the nose and pharynx due to contact with the drainage catheter and inconvenience due to the

\section{Karger'}


Case Reports in Gastroenterology
Case Rep Gastroenterol 2021;15:801-809

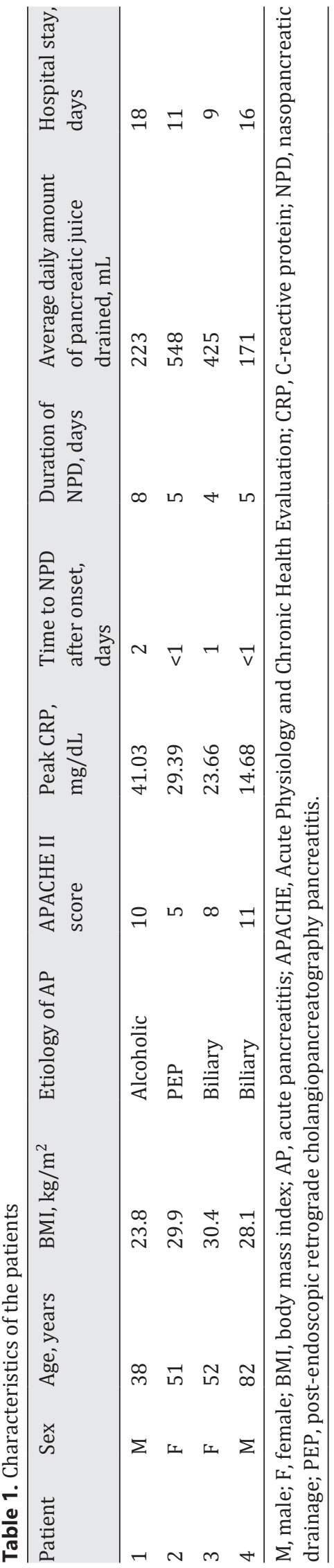




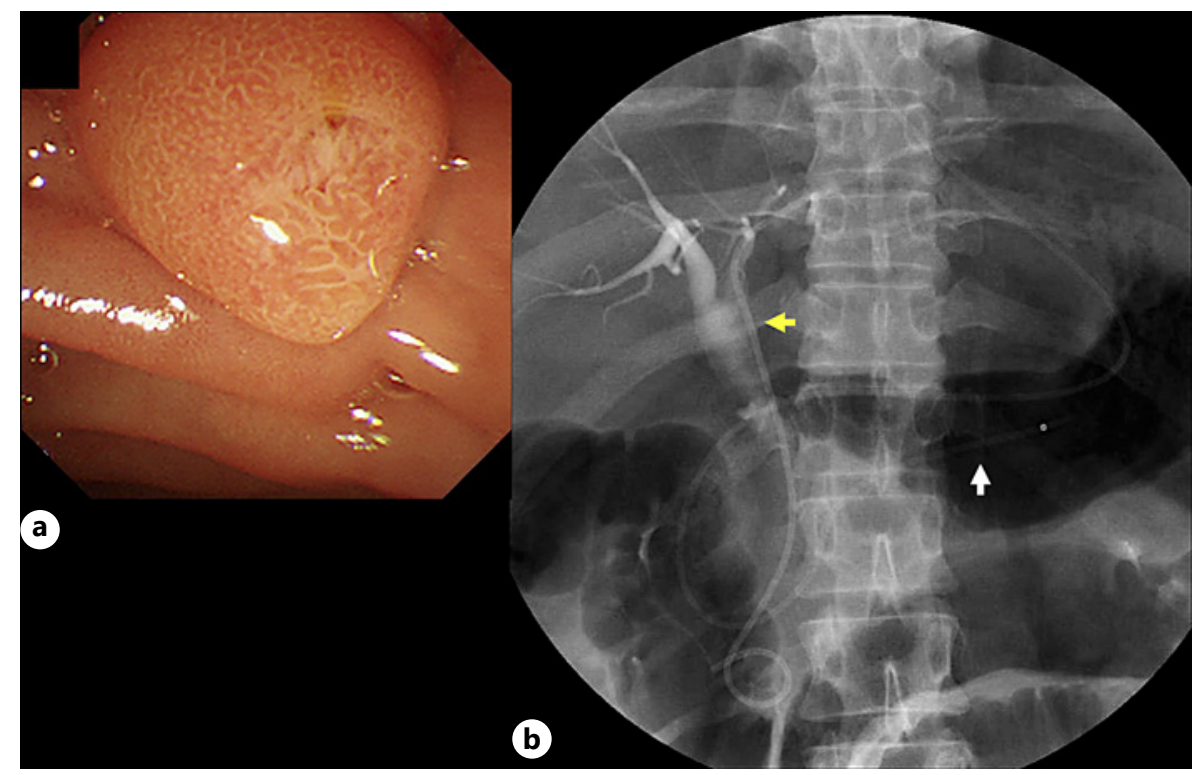

Fig. 1. a Endoscopy showed a swollen ampulla of Vater in the descending duodenum in patient 1 on day 3. b An NPD catheter (white arrow) and an endoscopic biliary stent (yellow arrow) were placed. NPD, nasopancreatic drainage.

catheter being outside the body. However, we thought that NPD provides decompression of the pancreatic duct by the drainage of pancreatic juice and contrast media more reliably than pancreatic duct stenting.

ERCP was performed using a duodenoscope (JF type 260v; Olympus Medical Systems, Tokyo, Japan), disposable cannula (V-system; Olympus Medical Systems), 0.025-inch hydrophilic guidewire (VisiGlide 2; Olympus Medical Systems), and nonionic isotonic contrast agent Iodixanol (Visipaque; GE Healthcare, Chicago, IL, USA). A 5-French plastic transpapillary biliary stent with double pigtails (Medi-Globe $\mathrm{GmbH}$, Rohrdorf, Bayern, Germany) was used for EBS. A straight 5-French plastic catheter without a flap (Gadelius Medical, Tokyo, Japan) was used for NPD (Fig. 1b). All procedures were carried out after obtaining written informed consent.

\section{Patient 1}

A 38-year-old man with severe upper abdominal pain presented to our hospital by an ambulance. He had diabetes, atopic dermatitis, depression, and a history of recurrent alcoholic AP. He drank about $90 \mathrm{~g}$ of ethanol and smoked 20 cigarettes daily. His vital signs in the emergency room were body temperature (BT) $36.4^{\circ} \mathrm{C}$, blood pressure (BP) $143 / 99 \mathrm{~mm} \mathrm{Hg}$, heart rate (HR) 99 beats/min, and respiratory rate (RR) 37 breaths/min. Laboratory tests showed serum amylase $263 \mathrm{U} / \mathrm{L}$, lipase $973 \mathrm{IU} / \mathrm{L}, \mathrm{C}$-reactive protein (CRP) $1.42 \mathrm{mg} / \mathrm{dL}$, white blood cell count (WBC) 10,800/ $\mathrm{LL}$, total bilirubin (T-Bil) $0.8 \mathrm{mg} / \mathrm{dL}$, aspartate aminotransferase (AST) $57 \mathrm{U} / \mathrm{L}$, alanine aminotransferase (ALT) $67 \mathrm{U} / \mathrm{L}$, alkaline phosphatase (ALP) 106 $\mathrm{U} / \mathrm{L}, \gamma$-glutamyl transpeptidase ( $\gamma$-GTP) $233 \mathrm{U} / \mathrm{L}$, and lactate dehydrogenase (LDH) 426 U/L. He was diagnosed with alcoholic AP. He underwent conservative treatment. Fentanyl was introduced for pain relief because other analgesics were insufficient. By day 3,>1,200 $\mu \mathrm{L}$ of fentanyl was required daily. At that time, abdominal CT showed marked swelling of the pancreas with extensive inflammation of peripancreatic adipose tissue (Fig. 2a, b). His vital signs were $\mathrm{BT} 38.8^{\circ} \mathrm{C}, \mathrm{BP} 133 / 88 \mathrm{~mm} \mathrm{Hg}$, HR 117 beats/min, and RR 42 breaths/min. Laboratory tests showed serum amylase $295 \mathrm{U} / \mathrm{L}$, lipase $581 \mathrm{IU} / \mathrm{L}$, CRP $32.16 \mathrm{mg} / \mathrm{dL}$, WBC 11,200/ $\mathrm{LL}$,

\section{Karger'}




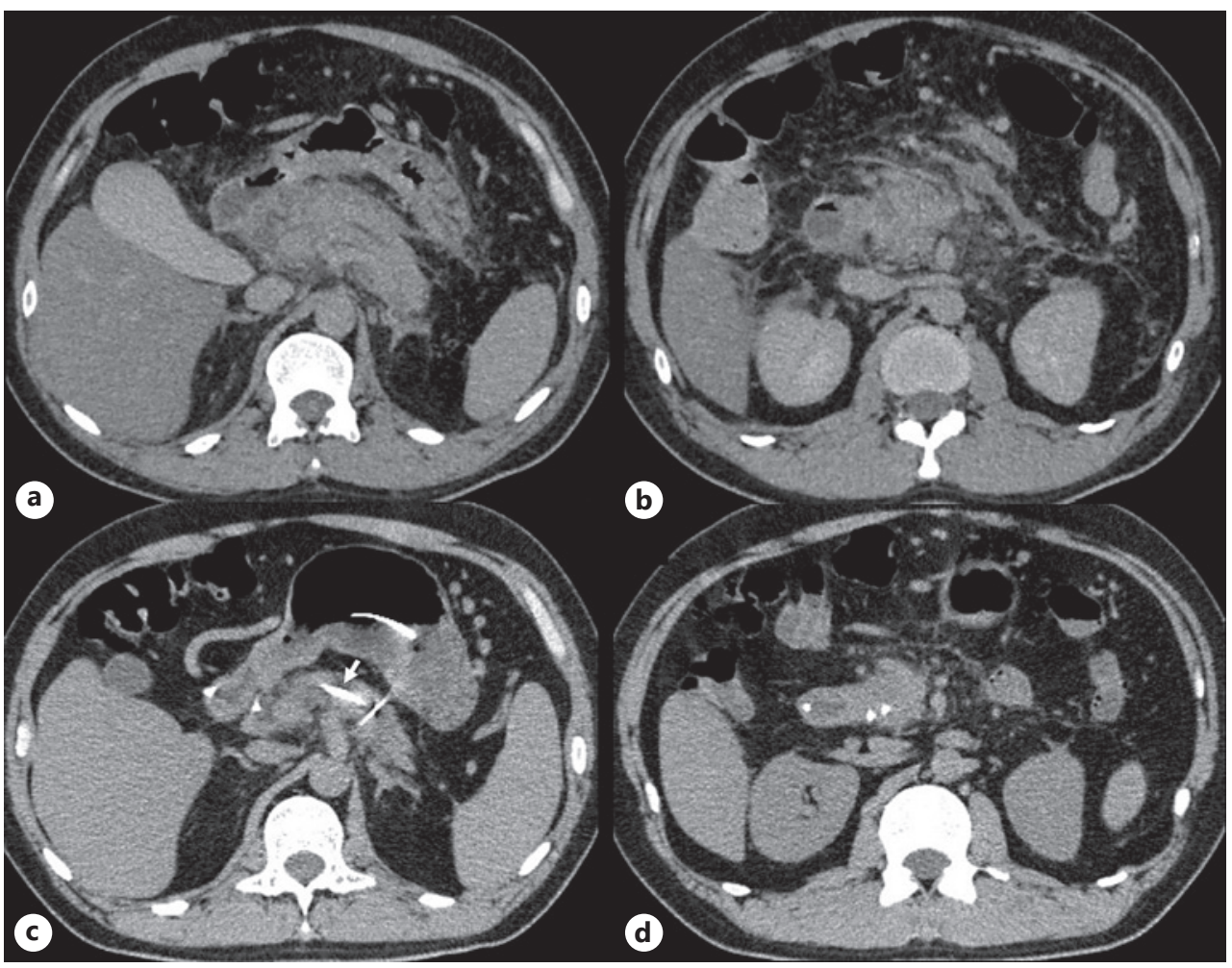

Fig. 2. Axial CT images of patient 1 on day 3 showed an edematous pancreatic body (a) and pancreatic head with marked peripancreatic inflammation (b). c, d Axial CT images of patient 1 on day 10 showed a marked decrease in pancreatic edema and peripancreatic inflammation. An NPD catheter was in place from day 3 to day 11 (c, arrow). CT, computed tomography; NPD, nasopancreatic drainage.

T-Bil $6.1 \mathrm{mg} / \mathrm{dL}$, direct bilirubin $4.4 \mathrm{mg} / \mathrm{dL}$, AST $178 \mathrm{U} / \mathrm{L}$, ALT $85 \mathrm{U} / \mathrm{L}$, ALP $93 \mathrm{U} / \mathrm{L}, \gamma$-GTP 323 U/L, and LDH 355 U/L. Sudden onset of jaundice, severe abdominal pain, and high-grade fever suggested the development of coexisting acute cholangitis secondary to an unknown biliary event. Acute cholangitis might have been the main cause of the sudden deterioration of his condition. Therefore, we decided to perform urgent ERCP on day 3. Endoscopy showed a swollen major duodenal papilla (Fig. 1a) that might occlude the bile duct and the pancreatic duct. We could not detect any biliary stones with cholangiography. Subsequently, EBS and NPD were performed (Fig. 1b). After drainage, the patient's condition rapidly improved, including a decrease in pain (Fig. 3). CT on day 10 showed a dramatic decrease in pancreatic swelling and peripancreatic inflammation (Fig. 2c, d). The patient developed pulmonary mycosis and was subsequently treated with antifungal drugs. The causal relationship between pulmonary mycosis and endoscopic treatment was unknown.

\section{Patient 2}

A 51-year-old woman presented to our department for treatment of common bile duct stones and liver dysfunction. Endoscopic treatment was performed. That night, she felt severe abdominal pain. Her vital signs were BT $37.7^{\circ} \mathrm{C}$, BP 149/98 mm Hg, HR 112 beats/min, and RR 18 breaths/min. Laboratory tests on the next morning showed serum amylase 1,869 U/L,

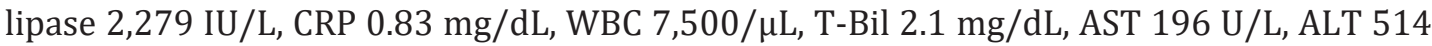
U/L, ALP $384 \mathrm{U} / \mathrm{L}, \gamma$-GTP $516 \mathrm{U} / \mathrm{L}$, and LDH $235 \mathrm{U} / \mathrm{L}$. Contrast-enhanced CT showed an extensive acute peripancreatic fluid collection, suggesting PEP with biliary obstruction that induced acute cholangitis. Edema at the ampulla of Vater, likely due to the last ERCP procedure, 


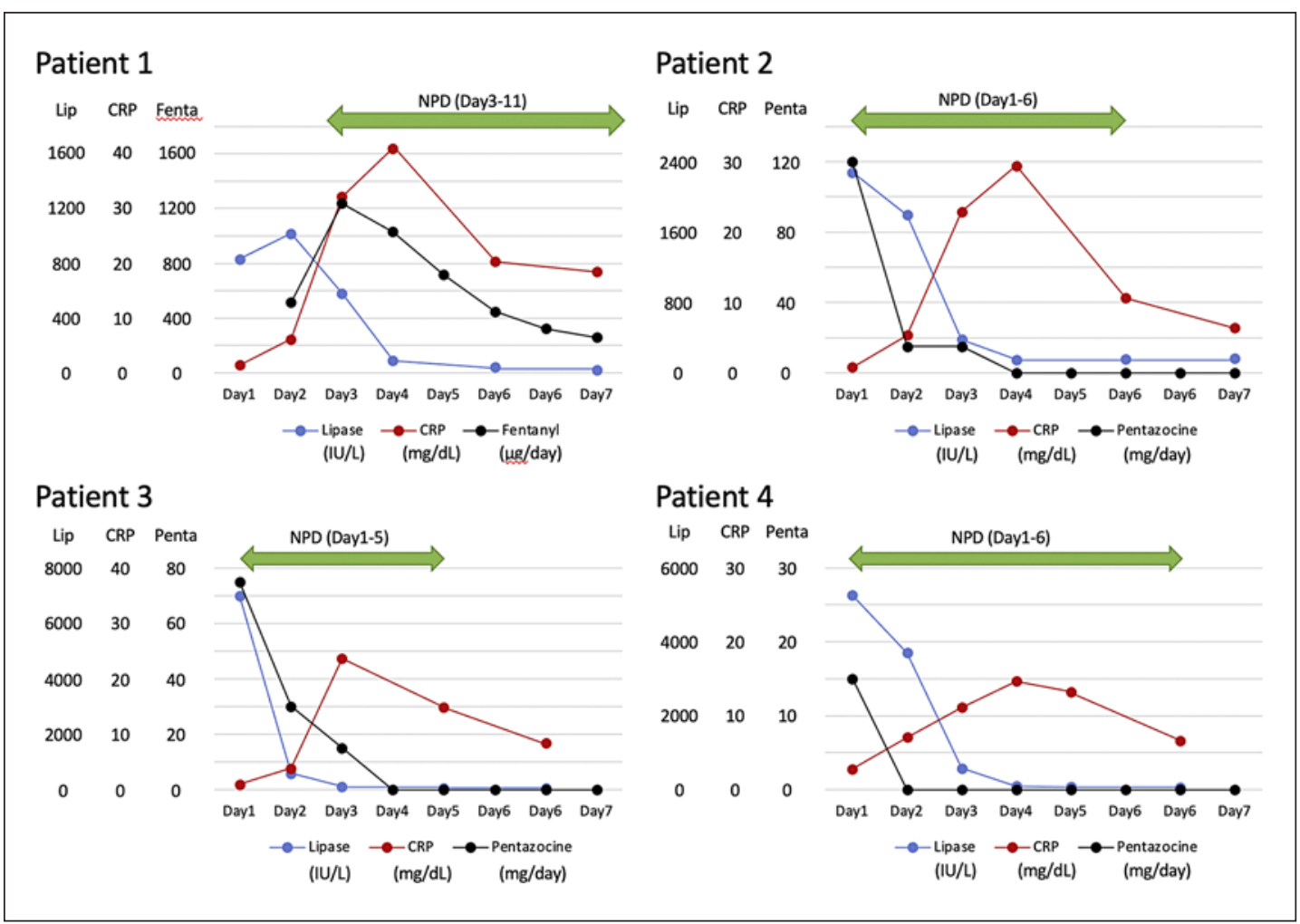

Fig. 3. Schematic diagram of the clinical course of patients 1-4. The NPD catheter was in place for 8 days in patient 1, 5 days in patient 2, 4 days in patient 3 , and 5 days in patient 4 . Blue dotted lines indicate serum lipase values, red dotted lines indicate CRP values, and black dotted lines indicate the amount of analgesics used each day. Fentanyl (patient 1) or pentazocine (patients 2-4) was used as the analgesic. Lip, lipase; CRP, C-reactive protein; Fenta, fentanyl; NPD, nasopancreatic drainage; Penta, pentazocine.

was predicted. She complained of severe continuous pain that required as much as $120 \mathrm{mg}$ of pentazocine over $18 \mathrm{~h}$. We decided to perform urgent re-ERCP for acute cholangitis. Endoscopically, the ampulla of Vater was extremely edematous, which might have occluded both the bile duct and the pancreatic duct. After the second ERCP with EBS and NPD, clinical symptoms resolved rapidly (Fig. 3). She did not have any systemic or local complications though previous CT images showed extensive peripancreatic inflammation. Contrastenhanced CT performed 3 weeks after PEP onset confirmed that there was neither necrosis nor the presence of a fluid collection.

\section{Patient 3}

A 52-year-old woman with severe upper abdominal pain and vomiting presented to our hospital by an ambulance. Her vital signs in the emergency room were BT $35.7^{\circ} \mathrm{C}, \mathrm{BP}$ $150 / 107 \mathrm{~mm} \mathrm{Hg}$, HR 87 beats/min, and RR 24 breaths/min. Laboratory tests showed serum amylase 3,045 U/L, lipase 7,000 IU/L, CRP $0.97 \mathrm{mg} / \mathrm{dL}, \mathrm{WBC}$ 19,200/ $\mu \mathrm{L}$, T-Bil 7.2 mg/dL, AST $472 \mathrm{U} / \mathrm{L}$, ALT $853 \mathrm{U} / \mathrm{L}$, ALP $161 \mathrm{U} / \mathrm{L}, \gamma$-GTP $434 \mathrm{U} / \mathrm{L}$, and LDH $417 \mathrm{U} / \mathrm{L}$. Contrast-enhanced CT showed an extensive acute peripancreatic fluid collection but no biliary stones. Clinical symptoms and laboratory data led to a diagnosis of obstructive acute cholangitis with a CT-negative biliary stone. We decided to perform urgent ERCP. After EBS and NPD, the severe pain was relieved (Fig. 3). Follow-up CT after discharge showed no local complications of AP. 


\section{Patient 4}

An 82-year-old man presented to our hospital's emergency room with severe upper abdominal pain. He had a history of acute thoracic aortic dissection surgery and cholecystectomy. His vital signs in the emergency room were BT $37.7^{\circ} \mathrm{C}, \mathrm{BP} 152 / 86 \mathrm{~mm} \mathrm{Hg}, \mathrm{HR} 112$ beats/min, and RR 30 breaths/min. Laboratory tests showed serum amylase 3,592 U/L, lipase 5,256 IU/L, CRP $2.80 \mathrm{mg} / \mathrm{dL}$, WBC 7,100/ $\mu \mathrm{L}$, T-Bil $2.86 \mathrm{mg} / \mathrm{dL}$, AST $382 \mathrm{U} / \mathrm{L}$, ALT 263 U/L, ALP $588 \mathrm{U} / \mathrm{L}, \gamma$-GTP $929 \mathrm{U} / \mathrm{L}$, and LDH $452 \mathrm{U} / \mathrm{L}$. We decided to perform urgent ERCP because CT and MRI findings suggested a biliary stone impacting the ampulla of Vater. As expected, we found an impacted biliary stone. We removed the stone and performed EBS and NPD. The patient's severe pain resolved after the procedure (Fig. 3). The clinical course was good despite the high risk of mortality from AP.

\section{Discussion}

This case series does not directly indicate the effectiveness of NPD for AP. All patients had dramatically rapid improvement in clinical symptoms, including pain relief. The improvement might have been primarily the effect of EBS for acute cholangitis. These 4 patients might have had mild AP with rapid improvement, which did not result in organ failure or local complications. However, our experience suggests that NPD catheter placement in these 4 patients did not exacerbate the pathology of existing AP. Therefore, NPD catheter placement might be safe when urgent ERCP is performed for a patient with AP. Furthermore, decompression of the pancreatic duct during the early phase of AP regardless of etiologies might improve the clinical course.

AP is an abdominal inflammatory disorder initiated by autodigestion due to activated pancreatic enzymes. Pancreatic duct obstruction, irrespective of the mechanism, leads to upstream blockage of pancreatic secretion, which in turn impedes exocytosis of zymogen granules containing digestive enzymes from acinar cells. Consequently, the lysosomal enzyme cathepsin B activates the conversion of the inactive digestive enzyme trypsinogen stored in zymogen granules into active trypsin. The resulting accumulation of trypsin can activate a cascade of digestive enzymes, leading to autodigestive injury [10]. Pancreatic duct obstruction can be caused by a stone impacting the ampulla of Vater in biliary AP [12], sphincter of Oddi spasm or edema at the ampulla of Vater due to cannulation trauma during ERCP [13], or protein plugs in alcoholic AP [10]. In an animal model of experimental pancreatitis, early decompression of the pancreatic duct improved the pathology of experimental biliary pancreatitis [11]. In the clinical setting, early pancreatic stent placement to decompress the pancreatic duct by restoring the outflow of pancreatic juices was associated with rapid PEP resolution [7]. However, it was recently reported that early placement of a pancreatic stent for necrotizing pancreatitis leads to a risk of retrograde gut bacterial infection in the sterile necrotic tissue [14]. On the other hand, NPD has a lower risk of retrograde gut bacterial infection than stent placement. A previous case series demonstrated that NPD for severe PEP is effective for early pain relief and might attenuate disease progression [15]. There is currently insufficient evidence that pancreatic duct decompression via NPD during the early phase of AP improves the clinical course.

According to the practice guidelines for AP, ERCP is only indicated for biliary AP $[1,2]$. On the other hand, the 2018 Tokyo Guidelines for acute cholangitis recommend endoscopic transpapillary biliary drainage via ERCP for acute cholangitis [3]. Two of the 4 patients had biliary AP with acute cholangitis. They had a true indication for ERCP according to AP guidelines. The remaining 2 patients were considered to have developed AP due to other causes and developed acute cholangitis simultaneously or secondary to AP. Patient 1 developed alcoholic AP first. Later, he had jaundice with direct bilirubin dominance, high-grade fever, and worsening severe abdominal pain on day 2 after admission. We speculated that some kind of

\section{Karger'}


biliary event had occurred, for example, acute cholangitis associated with a CT-negative biliary stone that had already passed when urgent ERCP was performed. Realistically, this was considered a rare case. However, even in the context of PEP and alcoholic AP, ERCP might be indicated for patients presenting with jaundice, fever, and abdominal pain when acute cholangitis could not be ruled out. Patient 2, who developed PEP after endoscopic biliary stone removal, developed concomitant acute cholangitis. Both AP and cholangitis were thought to be caused by pancreatic duct and biliary obstruction resulted from edema at the ampulla of Vater. This edema was due to cannulation trauma or mechanical stimulation of stone removal during the first ERCP. Our experience showed that NPD catheter placement does not worsen AP of various etiologies but rather might help improve the clinical course. More research is needed to clarify the safety and effectiveness of NPD for AP.

\section{Statement of Ethics}

This study was reviewed and approved by the institutional review broad, Approval No. (2020-2-217). Written informed consent was obtained from the patients involved in the study for publication of this case report and any accompanying images.

\section{Conflict of Interest Statement}

The authors have no conflicts of interest to declare.

\section{Funding Sources}

This case report has no funding sources.

\section{Author Contributions}

M.H. prepared the first draft of the manuscript. M.H., A.K., K.K., R.Y., A.T., and T.M. managed the patient and performed ERCP. K.E., T.K., and K.S. revised the manuscript. All authors approved the final manuscript.

\section{Data Availability Statement}

All data generated or analyzed during this study are included in this article. Further inquiries can be directed to the corresponding author. Data access requires proof of relevant ethics committee approval for the specified analysis only. Data will be limited to those required for a specific analysis to protect deanonymization.

\section{References}

1 Working Group IAP/APA Acute Pancreatitis Guidelines. IAP/APA evidence-based guidelines for the management of acute pancreatitis. Pancreatology. 2013 Jul-Aug;13(4 Suppl 2):e1-15.

2 Yokoe M, Takada T, Mayumi T, Yoshida M, Isaji S, Wada K, et al. Japanese guidelines for the management of acute pancreatitis: Japanese guidelines 2015. J Hepatobiliary Pancreat Sci. 2015 Jun;22(6):405-32. 
3 Mukai S, Itoi T, Baron TH, Takada T, Strasberg SM, Pitt HA, et al. Indications and techniques of biliary drainage for acute cholangitis in updated Tokyo guidelines 2018. J Hepatobiliary Pancreat Sci. 2017 Oct;24(10):537-49.

4 Cotton PB. Fifty years of ERCP: a personal review. Gastrointest Endosc. 2018 Auh;88(2):393-6.

5 Testoni PA, Mariani A, Aabakken L, Arvanitakis M, Bories E, Costamagna G, et al. Papillary cannulation and sphincterotomy techniques at ERCP: European Society of Gastrointestinal Endoscopy (ESGE) clinical guidelines. Endoscopy. 2016 Jul;48(7):657-83.

6 Mazaki T, Masuda H, Takayama T. Prophylactic pancreatic stent placementand post-ERCP pancreatitis: a systematic review and meta-analysis. Endoscopy. 2010 Oct;42(10):842-53.

7 Kerdsirichairat T, Attam R, Arain M, Bakman Y, Radosevich D, Freeman M. Urgent ERCP with pancreatic stent placement or replacement for salvage of post-ERCP pancreatitis. Endoscopy. 2014 Dec;46(12):1085-94.

8 Bhasin DK, Rana SS, Siyad I, Poddar U, Thapa BR, Sinha SK, et al. Endoscopic transpapillary nasopancreatic drainage alone to treat pancreatic ascites and pleural effusion. J Gastroenterol Hepatol. 2006 Jun;21(6):1059-64

9 Hanada K, Okazaki A, Hirano N, Izumi Y, Teraoka Y, Ikemoto J, et al. Diagnostic strategies for early pancreatic cancer. J Gastroenterol. 2015 Feb;50(2):147-54.

10 Lankisch PG, Apte M, Banks PA. Acute pancreatitis. Lancet. 2015 Jul;386(9988):85-96.

11 Runzi M, Saluja A, Lerch MM, Dawra R, Nishino H, Steer ML. Early ductal decompression prevents the progression of biliary pancreatitis: an experimental study in the Opossum. Gastroenterology. 1993 Jul;105(1):157-64.

12 Pandol SJ, Saluja AK, Imrie CW, Banks PA. Acute pancreatitis: bench to bedside. Gastroenterology. 2007 Mar; 132(3):1127-51.

13 Tryliskyy Y, Bryce GJ. Post-ERCP pancreatitis: pathophysiology, early identification and risk stratification. Adv Clin Exp Med. 2018 Jan;27(1):149-54.

14 Karjula H, Nordblad Schmidt P, Mäkelä J, Liisanantti JH, Ohtonen P, Saarela A. Prophylactic pancreatic duct stenting in severe acute necrotizing pancreatitis: a prospective randomized study. Endoscopy. 2019 Nov; 51(11):1027-34.

15 Kawaguchi S, Kikuyama M, Satoh T, Terada S. Use of nasopancreatic drainage for severe post-endoscopic retrograde cholangiopancreatography pancreatitis: a case series. Intern Med. 2018 Sep;57(18):2657-62. 